\title{
Temperature-Dependent Energy Transfer in Cadmium Telluride Quantum Dot Solids
}

\author{
Sander F. Wuister,* Rolf Koole, Celso de Mello Donegá, and Andries Meijerink \\ Condensed Matter and Interfaces, Debye Institute, Utrecht University, P.O. Box 80000, \\ 3508 TA Utrecht, The Netherlands
}

Received: November 16, 2004; In Final Form: January 28, 2005

\begin{abstract}
Efficiently luminescing colloidal CdTe quantum dots (QDs) were used for the preparation of monodispersed and mixed size QD solids. Luminescence spectra and decay times of the QD emission were measured as a function of temperature to study energy transfer (ET) processes in the QD solids. In the luminescence decay curves of the emission of the largest QDs (acceptors), a rise time of the luminescence signal is observed due to energy transfer from smaller QDs. Both the rise time (a measure for the energy transfer rate) and the luminescence decay time lengthen upon cooling. This is explained by the decreased dipole strength of the excitonic emission of the QDs in the solid due to the presence of a singlet and a lower lying triplet level. Studies of energy transfer in heteronuclear QD solids reveal that single-step ET dominates.
\end{abstract}

\section{Introduction}

Quantum dots (QDs) are widely studied because of their sizedependent electronic and optical properties. ${ }^{1,2}$ Recently, they have been used as building blocks for QD solids, which may find applications in, for example, QD solid lasers ${ }^{3}$ and QDbased light-emitting diodes (LEDs). ${ }^{4}$ Energy transfer (ET) between neighboring QDs is an important process in these applications. Assuming ET via dipole-dipole interaction, the ET rate scales with the distance via an $R^{-6}$ dependence. $^{5}$ Provided that there is spectral overlap (i.e., that the emission spectrum of the donor overlaps with the absorption spectrum of the acceptor), ET can occur between QDs in a QD solid. Since the exciton emission of the QDs has a large dipole moment at room temperature (reflected by the short radiative decay time), the ET rate can be fast if the distance between the QDs is sufficiently small. ${ }^{6-8}$

Kagan et al. reported ET between TOPO-capped CdSe QDs in an ordered QD solid. ${ }^{9,10}$ In a QD solid, ET from smaller QDs (larger band gap) to larger QDs (smaller band gap) can occur due to the size distribution. Recently, $\mathrm{CdSe}(\mathrm{ZnS})$ QDs were proposed for the preparation of a QD solid with engineered directional energy flows. ${ }^{5}$ In a QD solid with a layered energy gradient, ET rates between QDs of $10^{8}-10^{9} \mathrm{~s}^{-1}$ were reported. If the resonance conditions for ET are fine-tuned, rates close to $10^{10} \mathrm{~s}^{-1}$ can be obtained. ${ }^{6,7} \mathrm{Up}$ until now, the work on ET in QD solids has involved room-temperature measurements. In a few studies, low-temperature results were reported, ${ }^{9,10}$ but temperature-dependent studies of ET between QDs are still lacking.

In this paper, we report temperature-dependent studies of ET in QD solids, both homonuclear (only orange-emitting QDs) and heteronuclear (green- and orange-emitting QDs). QD solids were prepared from a solution of hexanethiol-capped CdTe QDs. For these high quality QDs, the luminescence decay curves are dominated by pure radiative decay. Time-resolved measurements on QD solids show pronounced rise times in the luminescence decay curves of the larger QDs (acceptors), reflecting the ET

* Author to whom correspondence should be addressed. Phone: +31 30-2532207. Fax: +31-30-2532403. E-mail: S. F.Wuister@phys.uu.nl. rate from the donors. Both the radiative decay rate and the ET rate decrease upon cooling, due to the smaller dipole moment of the excitonic emission at lower temperatures.

\section{Experimental Section}

The preparation of the CdTe QDs and of the CdTe QD solids was performed in a glovebox under a dry argon atmosphere.

Synthesis. Green-emitting $\left(\lambda_{\mathrm{em}} \approx 550 \mathrm{~nm}\right.$, quantum efficiency of $10 \%)$ and orange-emitting $\left(\lambda_{\mathrm{em}} \approx 600 \mathrm{~nm}\right.$, quantum efficiency of 27\%) DDA-capped QDs were prepared following a previously reported method (see ref 11 for a detailed description). Capping exchange with hexanethiol (HT) was achieved by adding $200 \mu \mathrm{L}$ of hexanethiol to $400 \mu \mathrm{L}$ of the crude solution of CdTe QDs in the TOP/DDA coordinating mixture. These particles were either dissolved in anhydrous chloroform or used for the preparation of QD solids.

Quantum dot solids were prepared by combining green- and orange-luminescing QDs in their original liquid matrix (TOP/ DDA) in the desired ratio of green to orange to a total volume of $400 \mu \mathrm{L}$. Capping exchange with hexanethiol was performed by addition of $200 \mu \mathrm{L}$ of hexanethiol. After 1 day to allow for capping exchange, this mixture was injected into $8 \mathrm{~mL}$ of anhydrous methanol to precipitate the QDs and remove most of the excess TOP, DDA, and hexanethiol. The precipitate was removed from the methanol solution, redissolved in a small amount of hexane, and slowly dried on a quartz substrate.

Apparatus. Emission spectra, recorded with a monochromator (Acton SP-300i, $0.3 \mathrm{~m}, 150$ lines $\mathrm{mm}^{-1}$ grating, blazed at $500 \mathrm{~nm}$, spectral resolution $1 \mathrm{~nm}$ ) fitted with a Princeton Instruments liquid-nitrogen-cooled charge-coupled device (CCD) camera, and luminescence decay curves were measured simultaneously using a PicoQuant laser $\left(\lambda_{\mathrm{ex}}=406 \mathrm{~nm}, 2.5 \mathrm{MHz}\right.$ repetition rate, 55 ps pulse width) as excitation source. For luminescence lifetime measurements, a monochromator (1350 lines $\mathrm{mm}^{-1}$ grating, blazed at $500 \mathrm{~nm}$, spectral resolution 10 $\mathrm{nm}$ ) in combination with a fast Hamamatsu photomultiplier tube (H5738P-01) was used for light detection. The luminescence decay curves were obtained by time-correlated single-photon counting (TCSPC) via time-to-amplitude conversion (TAC) with a Time Harp 100 computer card. The ratio of stop to start pulses 


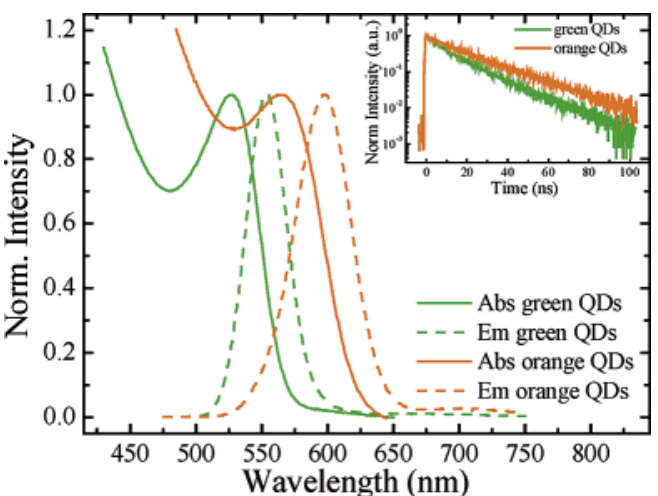

Figure 1. Normalized emission $\left(\lambda_{\mathrm{ex}}=400 \mathrm{~nm}\right)$ and absorption spectra of green- and orange-luminescing hexanethiol-capped CdTe QDs at room temperature. The inset shows luminescent decay curves (measured on the emission peak maximum, $\lambda_{\mathrm{ex}}=406 \mathrm{~nm}$ ) of both samples.

was kept low (below 0.05) to ensure good statistics. Temperature-dependent photoluminescence and lifetime measurements were carried out using a liquid helium flow cryostat equipped with a sample heater to stabilize the temperature at different temperatures between $4 \mathrm{~K}$ and room temperature. Samples were first cooled to $4 \mathrm{~K}$ and slowly heated to room temperature.

\section{Results and Discussion}

3.1. QD Solids Prepared from Homonuclear QDs. In this section, the room-temperature optical properties of the QDs used for the QD solids are discussed. Next, the temperature dependence of the emission and luminescence decay curves of orangeemitting CdTe QDs in solution and in a QD solid are discussed.

Figure 1 shows the absorption and emission spectra of a dilute solution of hexanethiol (HT)-capped CdTe QDs in chloroform for two different sizes. The green-emitting QDs (G-QDs) are $2.5 \mathrm{~nm}$ in diameter and show excitonic emission with a maximum at $555 \mathrm{~nm}$. The orange-emitting QDs (O-QDs) are $3.0 \mathrm{~nm}$ in size and emit at $600 \mathrm{~nm}\left(\lambda_{\max }\right)$. The inset shows the luminescence decay curves for both samples measured at the exciton peak maximum. The luminescence decay of the O-QDs shows a monoexponential behavior ${ }^{12}$ with a $\tau_{1 / e}$ of $19 \mathrm{~ns}\left(\tau_{1 / e}\right.$ is defined as the time at which the intensity reaches $1 / e$ of its initial intensity). This luminescence decay time is the radiative decay time for the CdTe QDs and shows the high quality of the O-QDs after capping exchange with hexanethiol. The decay of the G-QDs is not monoexponential and has a $\tau_{1 / e}$ of $11 \mathrm{~ns}$. This shorter decay is attributed to partial quenching of the exciton luminescence by surface and lattice defects. The fast initial luminescence decay reflects the presence of fast nonradiative processes that lower the quantum efficiency.

Figure 2 A shows the emission spectra of O-QDs (normalized to the emission maximum) in solution for two different temperatures ( 20 and $200 \mathrm{~K}$ ). A blue shift of the exciton peak is observed upon lowering the temperature, which is explained by the temperature dependence of the band gap of CdTe. ${ }^{13,14}$ The temperature dependence of the exciton decay time as function of the temperature has been studied in the last few years for CdSe QDs. ${ }^{15-17}$ The lowest level has a triplet character (also called dark state) while a slightly higher energy level has a singlet character (bright state). The energy difference between these two levels is a few millielectonvolts. ${ }^{15-17}$ At low temperatures, the excited-state population of the QDs will be mainly in the triplet state. The transition from this state to the ground state is spin-forbidden and characterized by a long decay time $(\sim 1 \mu \mathrm{s}) .{ }^{16}$ As the temperature increases, the singlet state becomes increasingly thermally populated (Boltzmann distribu-

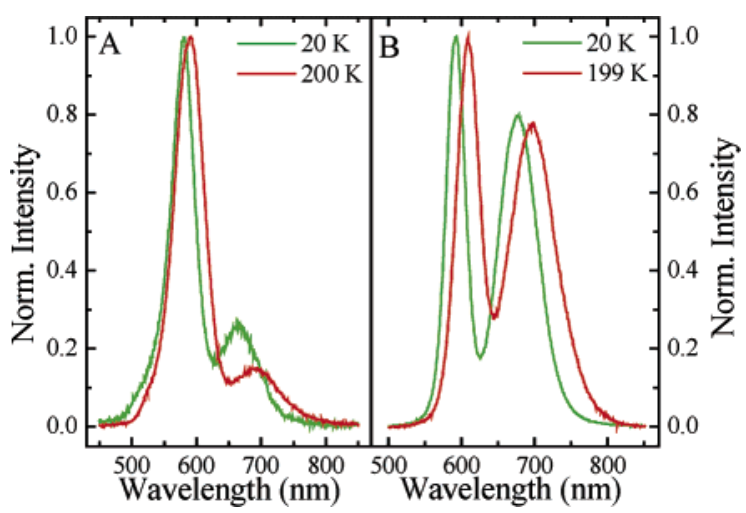

Figure 2. Normalized emission spectra $\left(\lambda_{\mathrm{ex}}=406 \mathrm{~nm}\right)$ of orangeluminescing hexanethiol-capped CdTe QDs dissolved in chloroform (A) and as a QD solid on quartz (B) recorded at $20 \mathrm{~K}$ (green curves) and $200 \mathrm{~K}$ (red curves).

tion). Since the transition from the singlet excited state to the ground state is spin-allowed, the dipole strength of the exciton emission will increase with increasing temperature. As a result, the radiative decay time decreases upon increasing the temperature. For CdTe QDs, a similar temperature dependence for the luminescence lifetime has been observed. ${ }^{18}$

Figure 2B shows the (normalized) emission spectra of a QD solid prepared from O-QDs recorded at 20 and $200 \mathrm{~K}$. The excitonic emission shifts from 582 to $594 \mathrm{~nm}(41 \mathrm{meV})$ at 20 $\mathrm{K}$ and from 594 to $609 \mathrm{~nm}(53 \mathrm{meV})$ at $200 \mathrm{~K}$ with respect to the exciton peak of the O-QDs in solution. Deconvolution of the spectra (by fitting two Gaussians) shows that the shift toward lower energy for the quantum dot solids is not due to the increased defect-related emission. The red shift is evidence for the occurrence of ET in the QD solid. The QD ensemble used to prepare the QD solid is not monodispersed in size $(\sim 15 \%$ size dispersion). ET occurs from donors to nearby accepting QDs with a slightly smaller band gap (larger size), and this results in a red shift of the ensemble exciton emission peak. In the absorption spectrum of the QD solid also, a small red shift with respect to the absorption spectrum in solution is observed for very small and monodispersed QDs due to interaction between neighboring QDs. ${ }^{19,20}$ For the presently studied (relatively large) QDs, this shift is too small to explain the shift observed in the emission spectra. The significant enhancement of the defect-related emission compared to the O-QDs in solution is also explained by ET. QDs with a defect act as a trap for the migrating energy. This leads to energy relaxation and the observation of defect-related emission. Figure 3 shows the luminescence decay curves at $20 \mathrm{~K}$ (part A) and $200 \mathrm{~K}$ (part B) of O-QDs dispersed in chloroform. Each graph in Figure 3 shows three decay curves recorded at the emission maximum (green curve), $20 \mathrm{~nm}$ blue-shifted from the emission maximum (purple curve), and $20 \mathrm{~nm}$ red-shifted from the emission maximum (red curve). The dependence of the luminescence decay behavior on the emission wavelength is weak, indicating that ET is absent in these diluted QD solutions due to the large distance between the QDs. A longer luminescence decay time is observed at $20 \mathrm{~K}$, which becomes increasingly shorter at higher temperatures $(200 \mathrm{~K})$. At $20 \mathrm{~K}$, a fast initial decrease in the luminescence decay curve is also observed. This is ascribed to a fast decay from the singlet level, similar to observations for CdSe QDs reported in ref 16.

Figure 3 shows the luminescence decay curves of a QD solid of O-QDs at $20 \mathrm{~K}$ (part C) and $200 \mathrm{~K}$ (part D). At $20 \mathrm{~K}$, a fast initial drop in intensity is observed for all emission wavelengths and is due to singlet emission before thermal equilibrium is 


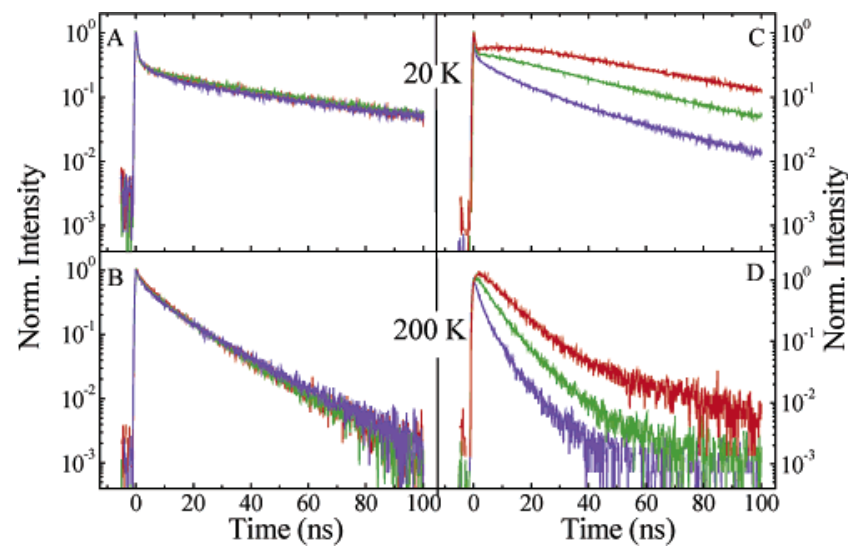

Figure 3. Normalized luminescence decay curves $\left(\lambda_{\mathrm{ex}}=406 \mathrm{~nm}\right)$ of orange-emiting hexanethiol-capped CdTe QDs dissolved in chloroform (left) and as a QD solid on quartz (right), recorded at 20 and $200 \mathrm{~K}$. The decay curves are measured at the exciton peak maximum (green curve), $20 \mathrm{~nm}$ red-shifted of the peak maximum (red curve), and 20 $\mathrm{nm}$ blue-shifted of the peak maximum (purple curve).

reached between the singlet and the triplet state. Contrary to the situation for the QDs in solutions, the luminescence decay curves are strongly dependent on the emission wavelength at both temperatures. The decay curves recorded at the shorter emission wavelength (in purple, corresponding to the smaller QDs) show a faster decrease in emission intensity than the decay curves recorded at longer wavelengths. In contrast to the fast decay for the smaller QDs, an increase in the luminescence intensity is observed in the curves for the emission at the longer wavelength (filled triangles, corresponding to the larger QDs). This is due to feeding by ET from smaller QDs to the larger QDs.

The strong wavelength dependence of the luminescence decay curves provides clear evidence that efficient ET occurs from the smaller O-QDs to the larger O-QDs in the CdTe QD solids. Qualitatively, the results are similar to time-resolved studies reported for CdSe QD solids. 5,6 The smallest QDs, emitting at the shortest wavelength, have a large number of neighboring acceptors to which ET can occur. As a result, these QDs have the shortest luminescence decay time. The largest O-QDs act as acceptors only and show, after a rise time due to feeding by ET from the smaller QDs, an exponential decay reflecting the radiative decay rate (Figure 3 parts $\mathrm{C}$ and $\mathrm{D}$, red curves). A quantitative analysis of the ET rates is complicated by several factors. The luminescence decay curves for the small QDs (the donors) are strongly nonexponential due an inhomogeneous distribution of acceptors around the donors. In addition, both nonradiative decay and ET to nonluminescent QDs may contribute to the observed decay curves of the luminescence for QD donors. The luminescence decay curves of the acceptors show an initial increase of the luminescence intensity with a rise time that is related to the transfer rate from the donating to the accepting QDs. Analysis of the rise time gives information on the transfer rate from the donor QDs. To obtain information on the temperature dependence of the ET rate, the decay curves of the acceptor emission (recorded $20 \mathrm{~nm}$ red-shifted of the emission maximum) were fitted to a biexponential function

$$
y(t)=y_{0}+A_{1} \mathrm{e}^{+\tau 1 / t}+A_{2} \mathrm{e}^{-\tau 2 / t}
$$

where $y_{0}$ is the offset (noise level), $\tau 1$ is the rise time of the luminescence intensity, and $\tau 2$ is the luminescence decay time. Figure 4 presents the decay times (dots, left-hand axis) and rise times (stars, right-hand axis) as a function of temperature. The

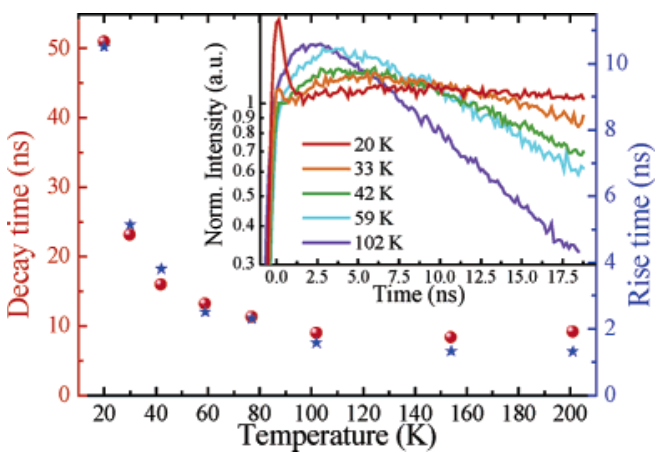

Figure 4. Luminescence decay $(\bullet)$ and rise time $(\star)$ of a QD solid on quartz, prepared from orange-luminescing hexanethiol-capped CdTe QDs recorded as function of temperature, measured $20 \mathrm{~nm}$ red-shifted of the excitonic peak maximum. The inset shows an enlargement of the normalized luminescence decay curves at different temperatures in the first $18 \mathrm{~ns}$.

inset of Figure 4 shows the first 18 ns of the luminescence decay curves of the acceptors, showing the rise time in more detail than in Figure 3. Figure 4 shows that the rise and decay times have the same temperature dependence. Over the full temperature range, the rise time is proportional to the decay time of the QD emission. This is an interesting observation. For ET via dipole-dipole interaction, the ET rate is expected to be proportional to both the donor and the acceptor oscillator strengths (see also below). In the present situation, the oscillator strength of the transition on the acceptor can be assumed to be constant as a function of temperature (the donor emission overlaps with the same excitation transitions on the acceptor). But the oscillator strength of the donor emission is strongly temperature-dependent and decreases at lower temperatures due to an increased population in the triplet excited state upon cooling. The fact that the experimentally observed ET rate scales with the (radiative) decay rate over the full temperature range gives evidence that the ET proceeds via a dipole-dipole interaction. ET via an exchange interaction mechanism can be excluded since for this mechanism the transfer rate is not influenced by the dipole strengths of the donor and acceptor transitions, ${ }^{21}$ but only by the overlap between the donor and acceptor wave functions.

From the values of the rise time, an estimate can be made of the ET rate. The rise time at $20 \mathrm{~K}$ is about $10 \mathrm{~ns}$, and it drops to approximately $1 \mathrm{~ns}$ at $200 \mathrm{~K}$. The rise time is much shorter than the radiative decay time, showing that ET has a higher probability than radiative decay. The corresponding ET rates are $10^{8} \mathrm{~s}^{-1}$ at $20 \mathrm{~K}$ and increase to $10^{9} \mathrm{~s}^{-1}$ at $200 \mathrm{~K}$. As indicated above, these numbers give the order of magnitude of the average ET rates from the donor QDs to multiple acceptors and cannot be taken as an accurate ET rate between one nearest neighbor donor-acceptor pair. Nevertheless, it is interesting to compare the experimentally observed transfer rate with the theoretically calculated transfer rate. Through the use of the Förster theory, the ET rate via dipole-dipole interaction can be derived to be $\mathrm{be}^{22}$

$$
k_{\text {Förster }}=\frac{Q_{\mathrm{D}}}{\tau_{\mathrm{D}}}\left(\frac{8.785 \times 10^{-25} I}{n^{4} R^{6}}\right)
$$

where $Q_{\mathrm{D}}$ is the dipole moment of the donor $(1), \tau_{\mathrm{D}}$ is the radiative decay time of the donor (19 ns), $n$ is the refractive index of the surrounding media (2.2), and $R$ is the distance between the dipoles. The overlap integral $I$ is given by

$$
I=\int_{0}^{\infty} \alpha_{\mathrm{A}}(\lambda) f_{\mathrm{D}}(\lambda) \lambda^{4} \mathrm{~d} \lambda
$$


where $\alpha_{\mathrm{A}}$ is the extinction coefficient of the acceptor $(1.5 \times$ $\left.10^{5} \mathrm{M}^{-1} \mathrm{~cm}\right), f_{\mathrm{D}}$ is the oscillator strength of the donor $(1.4 \times$ $10^{-12}$ ), and $\lambda$ is the absorption or emission wavelength (550 $\mathrm{nm})$. Through the use of these numbers, an ET rate at room temperature of $1.5 \times 10^{9} \mathrm{~s}^{-1}$ is calculated for ET between nearest neighbors at $3.5 \mathrm{~nm}$, the expected average distance between the O-QDs. The calculated ET rate is on the order of magnitude of the experimentally observed ET rate.

3.2. Heteronuclear QD Solids. In addition to using the polydispersity of a sample of QDs to observe ET, QD solids prepared from two sizes of QDs also were used to study the ET between the QDs. Two QD solids have been prepared with a molar ratio of G-QDs/O-QDs of 9:1 and 165:1. Temperaturedependent emission spectra are shown at two temperatures (20 and $200 \mathrm{~K}$ ) in Figure 5 for a QD solid with a G-QDs/O-QDs ratio of 9:1 (part A) and 165:1 (part B). In Figure 5A, three emission peaks are observed over the full temperature regime, a peak near $550 \mathrm{~nm}$ (the excitonic emission from the G-QDs), a peak near $590 \mathrm{~nm}$ (the excitonic emission from the O-QDs), and a broad band near $675 \mathrm{~nm}$, which is ascribed to the defectrelated emission of both G-QDs and O-QDs. The relative intensities of the green and orange emissions remain constant over the temperature range from 20 to $200 \mathrm{~K}$. This can be understood since both the radiative decay and the ET rate are dependent on the dipole strength of the exciton emission (as discussed above, see Figure 4). For the QD solid with the 165:1 ratio (Figure 5B), an emission peak near $550 \mathrm{~nm}$ is clearly visible, corresponding to the excitonic emission of the G-QDs. The broad peak near $650 \mathrm{~nm}$ is ascribed to the defect-related emission of the G-QDs. The exciton emission of the O-QDs is present as a weak shoulder between these two peaks. With temperature, this peak increases in intensity with respect to the exciton emission of the G-QDs, but the emission from the O-QDs is weak over the temperature range studied. For both samples, the intensity ratio of the excitonic and defect-related emission is almost independent of the sample temperature between 20 and $200 \mathrm{~K}$. The emission spectra of the mixed QD solids show that in the presently studied QD solids the ET is dominated by single-step energy transfer from donors to acceptors. In the 9:1 mixed QD solid, the acceptor concentration is high, $60-70 \%$ of the G-QD donors have one or more O-QDs as nearest neighbors, and efficient donor-acceptor ET is observed. The probability of finding one or more O-QDs as nearest neighbors is dependent on the coordination number in the QD solid. Since the studied QD solids are not expected to be ordered, the coordination number was varied between 8 (BCC packing) and 12 (FFC/HCP packing) to get an estimate for the probabilities.

In the 165:1 sample, the ET from the G-QD donors to the $\mathrm{O}-\mathrm{QD}$ acceptors is not probable by direct ET due to the low acceptor concentration; only 5-7\% of the G-QDs have an O-QD as nearest neighbor. The absence of a significant fraction of orange emission shows that ET via (multistep) energy migration over the G-QDs is not efficient. This is also reflected in the ratio of the intensities of the exciton peak of the G-QDs and the O-QDs in the 9:1 sample. On the basis of only radiative emission from the QDs, the intensity ratio of the G-QDs/OQDs, after correction for the QDs, should be 10:3. However, the emission of the O-QDs is much stronger than expected; an intensity ratio of approximately 10:17 is observed. From the difference in these two ratios, it was calculated that $50 \%$ of the G-QDs transfers its excitation energy to O-QDs. The probability of finding a G-QD that has one or more neighboring O-QDs is $60-70 \%$ in a QD solid with a G-QDs/O-QDs ratio of 9:1. This

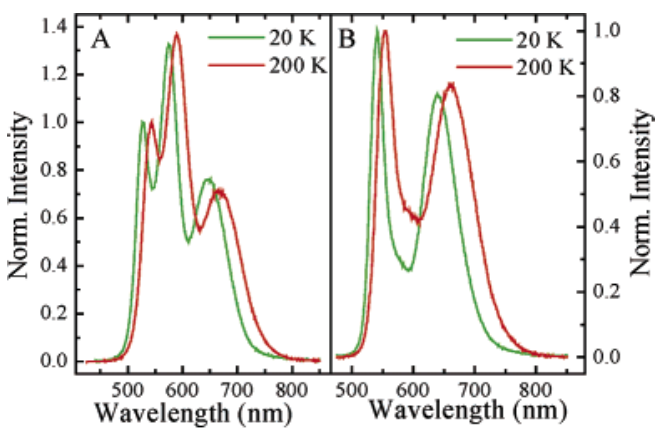

Figure 5. Normalized emission spectra $\left(\lambda_{\mathrm{ex}}=406 \mathrm{~nm}\right)$ of mixed hexanethiol-capped CdTe QD solids on quartz. The ratios of greenluminescing QDs to orange-luminescing QDs are 9:1 (A) and 165:1 (B).

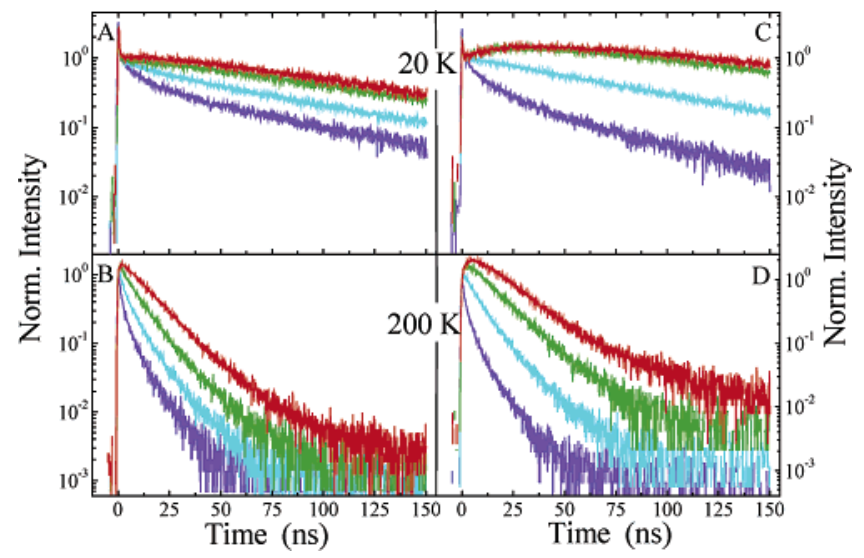

Figure 6. Normalized luminescence decay curves $\left(\lambda_{\mathrm{ex}}=406 \mathrm{~nm}\right)$ of mixed hexanethiol-capped CdTe QDs as QD solid on quartz, recorded at 20 and $200 \mathrm{~K}$. The ratios of G-QDs to O-QDs are 9:1 (A and B) and 165:1 ( $\mathrm{C}$ and $\mathrm{D})$. The decay curves are measured at the exciton peak maximum of the G-QDs (cyan curve), $20 \mathrm{~nm}$ red-shifted of this peak maximum (green curve), $20 \mathrm{~nm}$ blue-shifted of this peak maximum (purple curve), and at the exciton peak maximum of the O-QDs (red curve).

shows that nearly all of the G-QDs that have an O-QD as neighbor transfer their energy, indicating that ET between nearest neighbors is a very efficient process compared to radiative decay, as expected from the rates estimated above (at room temperature the ET rate is $2 \times 10^{9} \mathrm{~s}^{-1}$, and the radiative decay rate is $5 \times 10^{7} \mathrm{~s}^{-1}$ ).

Figure 6 shows the luminescence decay curves of the QD solid with a G-QD/O-QD ratio of 9:1 at two selected temperatures, $20 \mathrm{~K}$ (part A), and $200 \mathrm{~K}$ (part B), and of the QD solid with a G-QD/O-QD ratio of 165:1 at $20 \mathrm{~K}$ (part C) and $200 \mathrm{~K}$ (part D) In each graph, four curves are depicted, all recorded at different emission wavelengths, at the excitonic peak maximum of the G-QDs (cyan curve), $20 \mathrm{~nm}$ blue-shifted (purple curve) or red-shifted (green curve) of the peak maximum, and at the excitonic peak maximum of the O-QDs (red curve). A similar wavelength dependence of the decay curves is observed as that in Figure 3; the luminescence decay is faster at shorter wavelengths and becomes faster as the emission wavelength (i.e., the particle size) increases. This shows that ET occurs in both heteronuclear QD solids. After comparison of the luminescence decay curves of the two heteronuclear QD solids, interesting differences are observed. At $200 \mathrm{~K}$, the decay curves recorded $20 \mathrm{~nm}$ blue-shifted from the G-QDs exciton peak maximum are identical, showing that the ET rate of the smallest particles in both solids is comparable. For the O-QDs (red curves), the rise time is more pronounced in the sample with a G-QDs/O-QDs ratio of 165:1. The rise time in the decay curves 
of these QDs reflects their role as acceptors. The main difference between the two heteronuclear QD solids is the concentration of O-QD. For the QD solids with a G-QDs/O-QDs ratio of 9:1, the higher $\mathrm{O}-\mathrm{QD}$ concentration gives rise to a probability of finding a larger O-QD as nearest neighbor for an O-QD of 30$35 \%$, while for the QD solid with a G-QDs/O-QDs ratio of 165:1 this probability is $2-4 \%$. ET between two O-QDs will diminish the rise time of the (smaller) O-QDs. Since the probability of finding one or more O-QDs as nearest neighbors is much larger in the QD solid with a G-QDs/O-QDs ratio of 9:1, the rise time of the O-QDs is less pronounced due to ET between O-QDs. In the QD solid with a G-QDs/O-QDs ratio of 165:1, the ET between O-QDs is negligible. For this QD solid, the rise in intensity observed in the decay curve of the O-QDs reflects the feeding by G-QDs.

\section{Conclusions}

QD solids are prepared from efficiently luminescing colloidal CdTe QDs. Energy transfer (ET) is observed in both homonuclear and heteronuclear QD solids from smaller to larger QDs. Both the radiative decay rate and the ET rate are dependent on temperature and decrease upon cooling. The rise times in the luminescence decay curves of the larger (energy accepting) QDs reflect the ET rate. Over the temperature range studied here, the ET rates are observed to be proportional to the radiative decay rate that is consistent with a dipole-dipole mechanism for ET. Studies of ET in heteronuclear QD solids show that ET is dominated by a single-step ET process.

Acknowledgment. This work was financially supported by Utrecht University within the "Breedte-Strategie" program "Physics of Colloidal Matter". The experimental expertise of Veronique Gevaerts and Marijn Holman is gratefully acknowledged.

\section{References and Notes}

(1) Alivisatos, A. P. J. Phys. Chem. 1996, 100, 13226.

(2) Brus, L. E. J. Phys. Chem. 1986, 90, 2555

(3) Klimov, V. I.; Mikhailovsky, A. A.; Xu, S.; Malko, A.; Hollingsworth, J. A.; Leatherdale, C. A.; Eisler, H.; Bawendi, M. G. Science 2000, 290, 314.

(4) Coe, S.; Woo, W.-K.; Bawendi, M. G.; Bulovic, V. Nature 2002, 420,800 .

(5) Crooker, S. A.; Hollingsworth, J. A.; Tretiak, S.; Klimov V. I. Phys. Rev. Lett. 2002, 89, 186802.

(6) Achermann, M.; Petruska, M. A.; Crooker, S. A.; Klimov V. I. J. Phys. Chem. B 2003, 107, 13782.

(7) Franzl, T.; Koktysh, D. S.; Klar, T. A.; Rogach, A. L.; Feldmann, J.; Gaponik, N. Appl. Phys. Lett. 2004, 84, 2904.

(8) Förster, T. Naturwissenschaften 1946, 33, 166.

(9) Kagan, C. R.; Murray, C. B.; Nirmal, M.; Bawendi, M. G. Phys. Rev. Lett. 1996, 76, 1517.

(10) Kagan, C. R.; Murray, C. B.; Bawendi, M. G. Phys. Rev. B 1996, $54,8633$.

(11) Wuister, S. F.; Van Driel, F.; Meijerink, A.; Phys. Chem. Chem. Phys. 2003, 5, 1253.

(12) Wuister, S. F.; Swart, I.; Van Driel, F.; Hickey, S. G.; de Mello Donegá, C. Nano Lett. 2003, 3, 503.

(13) Suyver, J. F.; van der Beek, T.; Wuister, S. F.; Kelly, J. J.; Meijerink. A. Appl. Phys. Lett. 2001, 79, 4222.

(14) Varshni, Y. P. Physica 1967, 34, 149.

(15) Nirmal, M.; Norris, D. J.; Kuno, M.; Bawendi, M. G.; Efros, Al. L.; Rosen, M. Phys. Rev. Lett. 1995, 75, 3728.

(16) Labeau, O.; Tamarat, P.; Lounis, B. Phys. Rev. Lett. 2003, 90 , 257404-1.

(17) Efros, Al. L.; Rosen, M.; Kuno, M.; Nirmal, M.; Norris, D. J.; Bawendi M. G. Phys. Rev. B 1996, 54, 4843.

(18) de Mello Donegá, C.; Kleijer, C.; Meijerink, A. Unpublished work.

(19) Artemyev, M. V.; Woggon, U.; Jaschinski, H.; Gurinovich, L. I.; Gaponenko, S. V. J. Phys. Chem. B 2000, 104, 11617.

(20) Döllefeld, H.; Weller, H.; Eychmüller, A. J. Phys. Chem. B 2002, 106, 5604 .

(21) Blasse, G.; Grabmaier, B. C. Luminescent Materials; SpringerVerlag: New York, 1994.

(22) Scholes, G. D. Annu. Rev. Phys. Chem. 2003, 54, 57. 